\title{
GTNP Breeding Bird Monitoring Project 2001 SEASON
}

\author{
M. L. CODY $\downarrow$ DEPARTMENT OF BIOLOGY \\ UNIVERSITY OF CALIFORNIA \ LOS ANGELES
}

\section{$\downarrow$ OVERVIEW: GTNP BREEDING} BIRD MONITORING PROJECT:

1. Following initial independent work by M. L. Cody and by funding from NPS, we instigated a scheme for long-term monitoring of breeding land bird populations in a wide variety of habitats representative of the northern Rockies and the Greater Yellowstone Ecosystem (GYE). Census sites are located almost entirely within Grand Teton National Park, where a broad range of representative vegetation types is accessible within close geographic proximity.

2. 30 monitoring sites are established within and adjacent to the park in pristine habitat. Sites range from the Jackson Hole lowlands to subalpine and alpine sites, from meadow, sagebrush and marshland, through willow scrub, cottonwood and aspen woodlands, to lodgepole pine and spruce-fir forests. Some sites have a monitoring history of $>30 \mathrm{y}$; others were established in the mid-1990's.

3. The location and accessibility of the study sites permits all to be regularly and repeatedly censused during the short (6-week) breeding season. Census sites are standardized in area (510 ha in size) and mapped in detail (topographic features, vegetation). Census schedules, timing, and methodological protocols are established, and allow for controlled inter-site and inter-year comparisons in breeding bird populations, species composition, and densities.

\section{SEASON: COVERAGE AND PARTICIPANTS}

Participants. Four persons participated in the 2001 census effort, as listed below:

Anna Chalfoun

Resouce Management

GTNP, Moose, WY

Martin L. Cody

Dept. Organismic Biology, Ecology \& Evolution

University of California

Los Angeles, CA 90095-1606

PH: 310-825-1327; FX: 310-206-3987; EM:

mlcody@ucla.edu

Matt Killebrew

P.O. Box 284

Moose, WY 83012

PH: 307/734/0652; EM: Matt_Killebrew@nps.gov

Sue Wolff

PO Box 91

Moose, WY 83102

307-739-3464; EM: susan_wolff@nps.gov

Site Coverage

Eighteen of the 30 monitoring sites were assessed in 2001. Coverage was continued on those sites with the longest monitoring history, which in some cases covers several decades; coverage also spanned the full habitat range of GTNP's monitoring sites (see Table 1). 
The last column of the table indicates the sites censused the 2001 season; the selection continues an unbroken continuity at important "flagship" sites and also extends coverage of some less routinely censused areas. Overall, most of the 30 sites have been censused over about a decade.

\section{The Y2001 Censuses}

The list of breeding species seen at the monitoring sites (Appendix A) now stands at 152 . Y2001 was a dry year; a review of the climate data from the Moran weather station is summarized as follows. Over the last $50 \mathrm{y}$, precipitation AUG-JUL (basically the 12 months preceding a breeding census survey) averaged $606 \mathrm{~mm}$. Precipitation 2000-01 was well below average $(466 \mathrm{~mm})$, while the annual mean temperature was very close to the long-term mean.

Breeding densities of representative common species are summarized in Figure 1 Site 225. In this figure, sites grouped into "meadow", "willow-aspen-cottonwood" and "pine-spruce-fir" categories respectively depict the densities of representative species plotted as functions of time. In Figure 2, the breeding sparrows in sites near Jackson Lake Junction are shown in the same axes.
Monitoring data are now sufficiently extensive so that further analyses are possible. The coefficient of variation (CV) is reasonably evaluated over a ten-year stretch, and the relation between breeding density $\mathrm{CV}$ and migration strategy addressed. Figure 3 (from Cody, 2002) shows that, contrary perhaps to the most obvious hypothesis that long-distance migrants will be more variable in breeding density year-to-year, the opposite is the case, with least variation seen in species wintering further from GTNP. The relation, while not statistically significant, is nevertheless suggestive and interesting. 
Table 1. Cody, M. L. 2002. Seasonal bird traffic between Jackson Hole, Wyoming, and western Mexico. Proc. Partners in Flight Asilomar Conference, USFS Gen. Tech. Rep. (in press).

COVERAGE OF GTNP BREEDING BIRD MONITORING SITES, 1966-2001

\begin{tabular}{|c|c|c|c|c|c|c|c|c|c|c|c|c|}
\hline Site/Censused & $66 \quad 91$ & 92 & 93 & 94 & 95 & 96 & 97 & 98 & 99 & 0 & 1 & $\begin{array}{l}\text { Total } \\
\text { Yrs }\end{array}$ \\
\hline 1:Wolf Rch & \multirow{8}{*}{1} & \multirow{3}{*}{$\begin{array}{l}1 \\
1\end{array}$} & \multirow{3}{*}{$\begin{array}{l}1 \\
1\end{array}$} & 1 & 1 & 1 & \multicolumn{3}{|l|}{1} & \multicolumn{2}{|l|}{1} & 7 \\
\hline 2:JLJ Sedge & & & & 1 & 1 & 1 & 1 & 1 & 1 & 1 & 1 & 10 \\
\hline 3:Elk Refuge & & & & 1 & 1 & 1 & 1 & 1 & 1 & 1 & & 7 \\
\hline 4:JLJ Gr-sage & & 1 & 1 & 1 & 1 & 1 & 1 & 1 & 1 & 1 & 1 & 12 \\
\hline 5: Ant.Flats & & & & & 1 & 1 & 1 & & 1 & 1 & & 5 \\
\hline 6: Airpt Sage & & & 1 & 1 & 1 & 1 & 1 & 1 & & 1 & 1 & 8 \\
\hline 7: 2OL Meadow & & & 1 & 1 & 1 & 1 & 1 & 1 & 1 & 1 & 1 & 9 \\
\hline 8:Triangle $\mathrm{X}$ & & & & & & 1 & 1 & & & 1 & & 3 \\
\hline 9: RKO & & & 1 & 1 & 1 & 1 & 1 & 1 & 1 & 1 & 1 & 9 \\
\hline 10:JLJ Will & 1 & 1 & 1 & 1 & 1 & 1 & 1 & 1 & 1 & 1 & 1 & 12 \\
\hline 11: Oxbow & & & 1 & 1 & 1 & 1 & 1 & 1 & 1 & 1 & 1 & 9 \\
\hline 12: Elk Rch W & 1 & 1 & 1 & 1 & 1 & 1 & 1 & & & & & 7 \\
\hline 13: Elk Rch E & 1 & 1 & 1 & 1 & 1 & 1 & 1 & 1 & & 1 & & 9 \\
\hline 14: Cow Lk & 1 & 1 & 1 & 1 & 1 & 1 & 1 & & 1 & & 1 & 9 \\
\hline 15: Spread Ck & & 1 & 1 & 1 & 1 & 1 & 1 & 1 & & 1 & & 8 \\
\hline 16: Schwabch & & 1 & 1 & 1 & 1 & 1 & 1 & & 1 & & 1 & 8 \\
\hline 17: L. Granite & & & & & 1 & 1 & 1 & & & 1 & & 4 \\
\hline 18: Timbered Is & & & & 1 & 1 & 1 & 1 & 1 & 1 & & 1 & 7 \\
\hline 19: AMK & & 1 & 1 & 1 & 1 & 1 & 1 & 1 & 1 & 1 & 1 & 10 \\
\hline 20: TaggartLk & & & & 1 & 1 & 1 & 1 & 1 & & & 1 & 6 \\
\hline 21: Signal Mt & & 1 & 1 & 1 & 1 & 1 & 1 & 1 & 1 & 1 & & 9 \\
\hline 22: Spaulding & & & & & 1 & 1 & 1 & & & & 1 & 4 \\
\hline 23: LizardCk & & 1 & 1 & 1 & 1 & 1 & 1 & & & & 1 & 7 \\
\hline 24: Bradley & & & & 1 & 1 & 1 & 1 & & & & 1 & 5 \\
\hline 25:JennyLk & & & 1 & 1 & 1 & 1 & 1 & 1 & 1 & 1 & 1 & 9 \\
\hline 26:GranSpruce & & & & 1 & 1 & 1 & 1 & & 1 & & & 5 \\
\hline 27:Rendezvous & & & & 1 & 1 & 1 & 1 & & 1 & & & 5 \\
\hline 28:CodyBowl & & & & 1 & 1 & 1 & 1 & & 1 & 1 & 1 & 7 \\
\hline 29:HeronLk & & & & & 1 & 1 & 1 & & 1 & 1 & & 5 \\
\hline 30:Blacktail & & & & & & 1 & 1 & & 1 & 1 & 1 & 5 \\
\hline & & & & & & & & & & & & AVE \\
\hline Total Sites: & 2 & 12 & 17 & 24 & 28 & 30 & 30 & 15 & 19 & 20 & 18 & 7.52 \\
\hline
\end{tabular}


Figure 1.

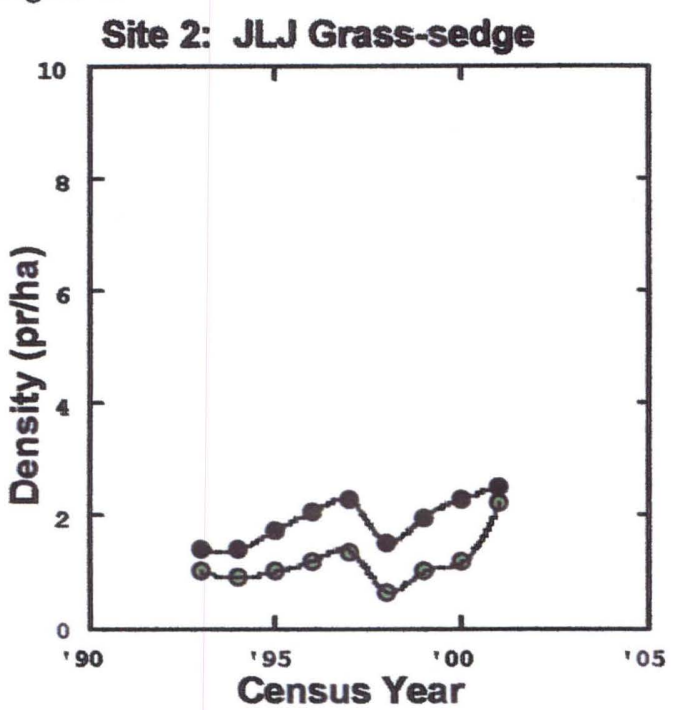

Site 4: JLJ Grass-sage

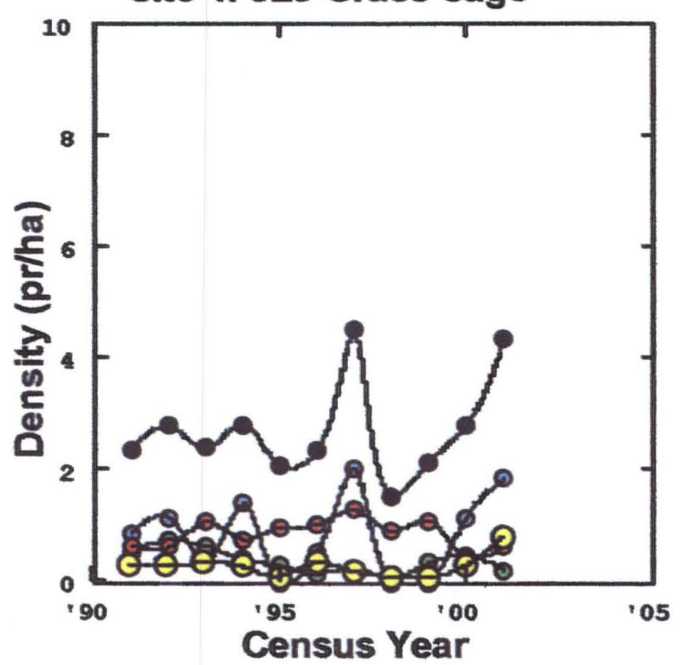

Site 7: Two Ocean Lake Meadow

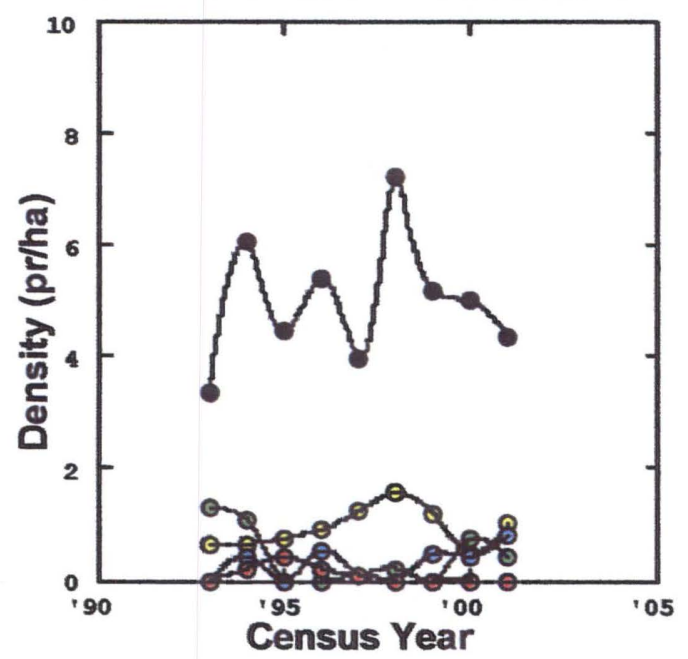

Site 3: Elk Refuge Wet Meadow

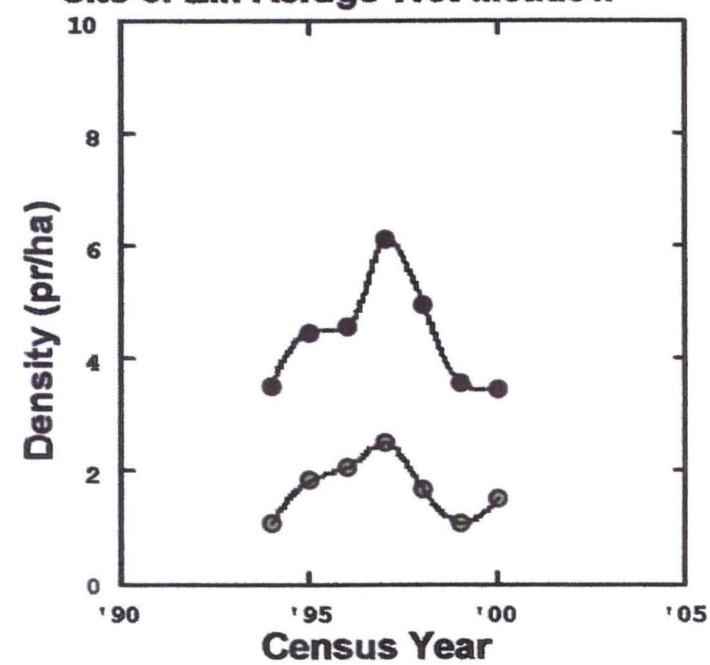

Site 6: Airport Sage

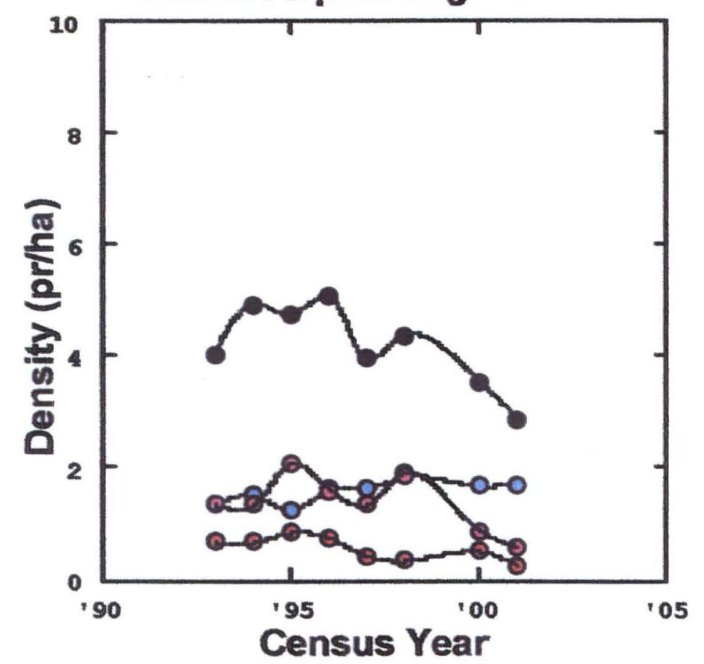

- All species

- Savannah Sparrow

- Vesper Sparrow

O Brewer"s Sparrow

O Wh.cr.sparrow 

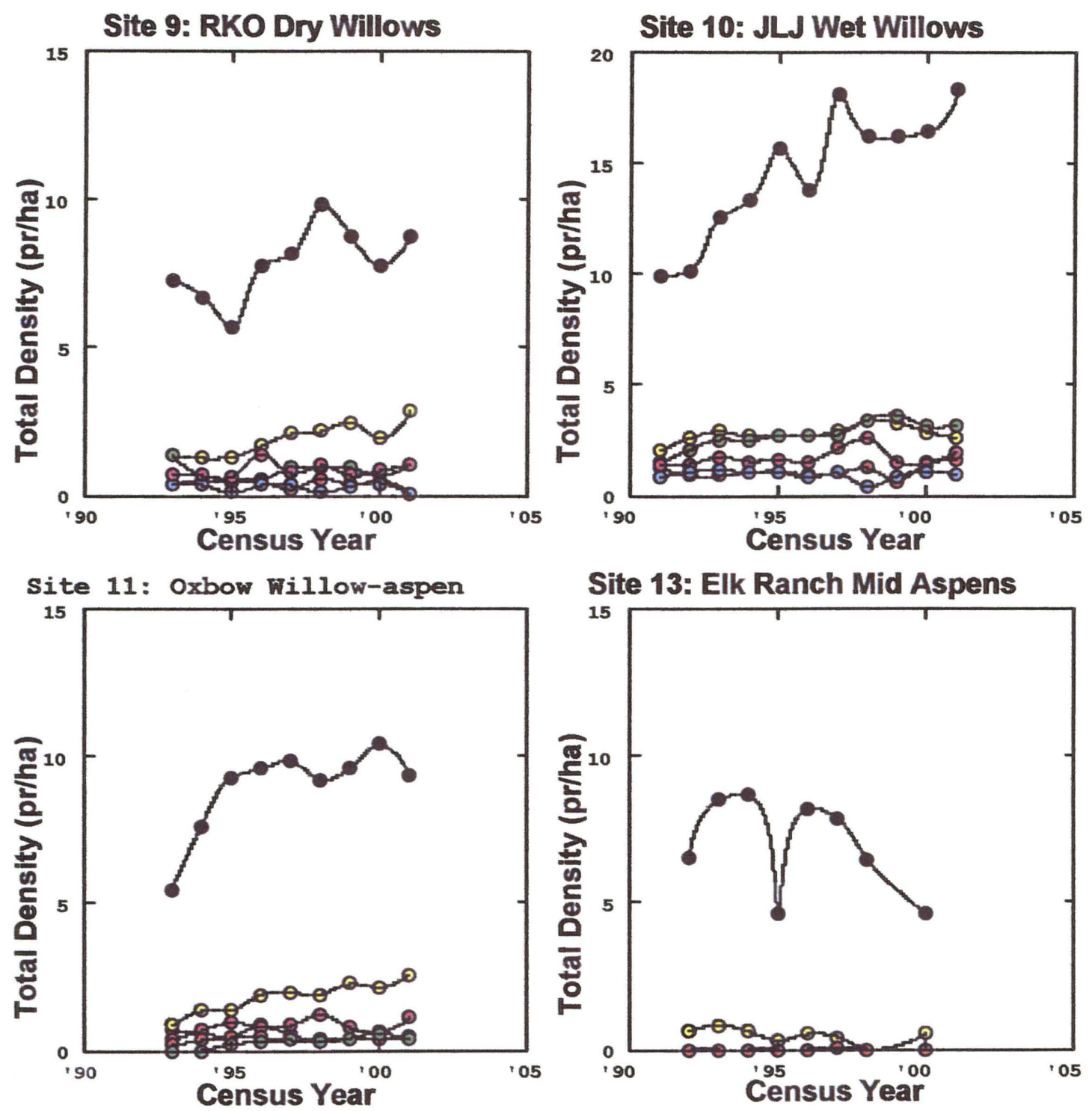

Site 15: Spread Ck Cottonwoods

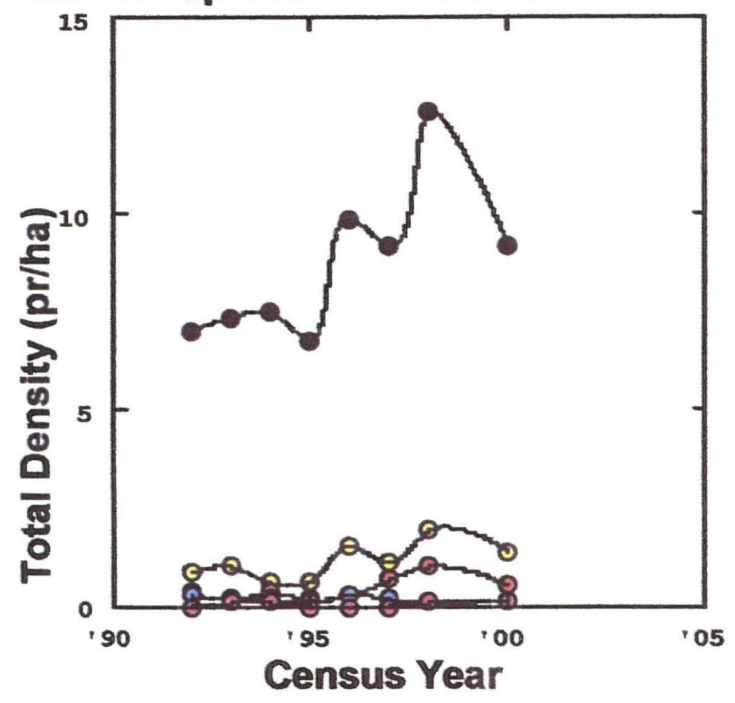

Total Bird Density

O Yellow Warbler

○ C. Yellowthroat

O Lincoln's Sparrow

O Fox Sparrow

- Song Sparrow 

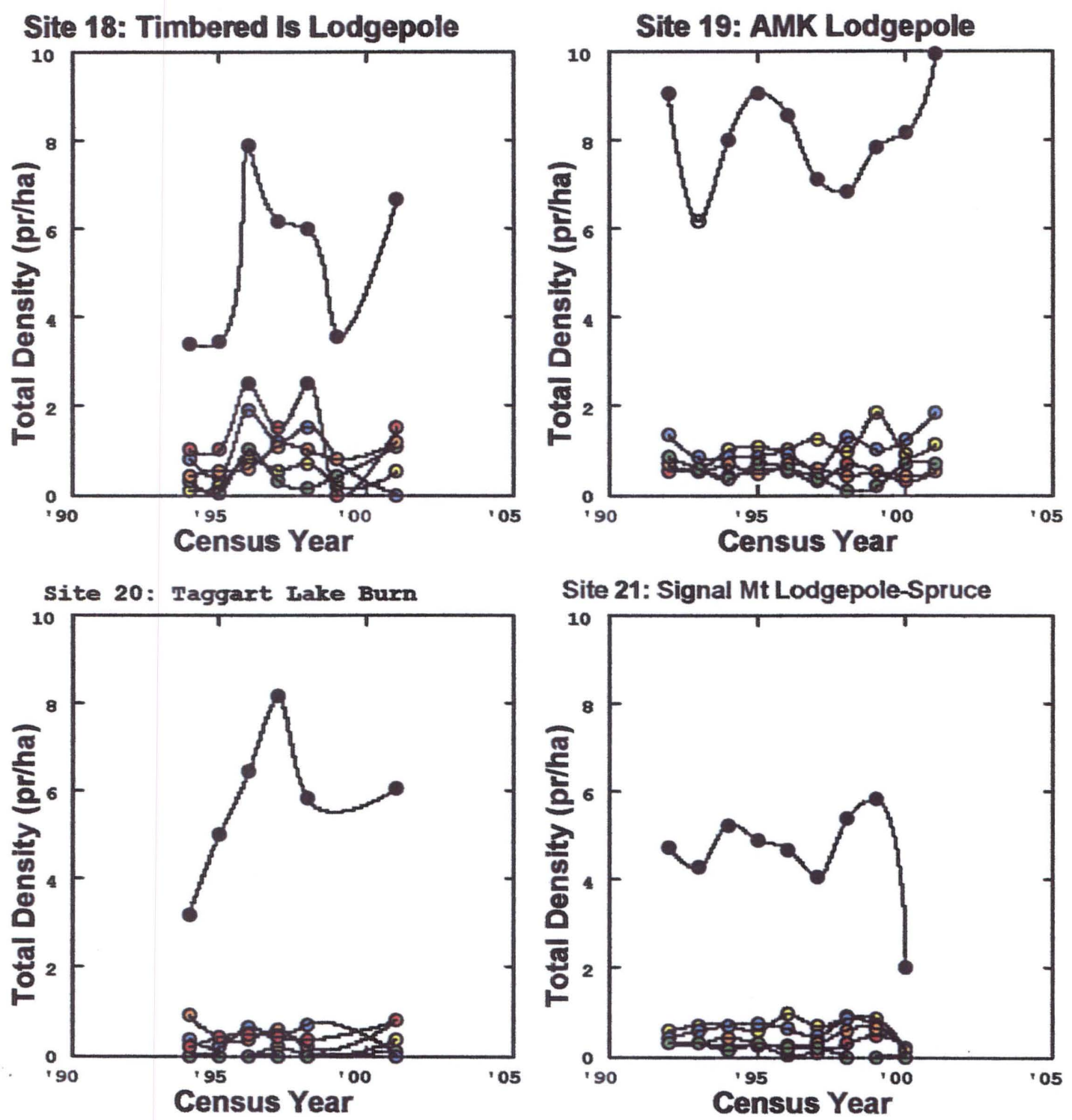

Site 25: Jenny Lake Spruce-Fir

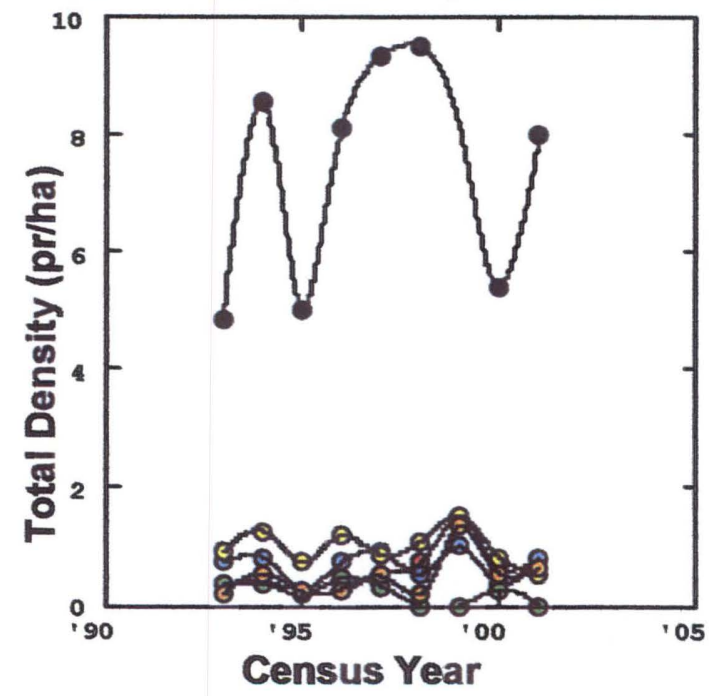

Total Bird Density

O Yellow-rumped Warbler

O Dark-eyed Junco

O Chipping Sparrow

O Ruby-crowned Kinglet

- American Robin 
Figure 2.

JACKSON LAKE JUNCTION: SPARROW DENSITIES
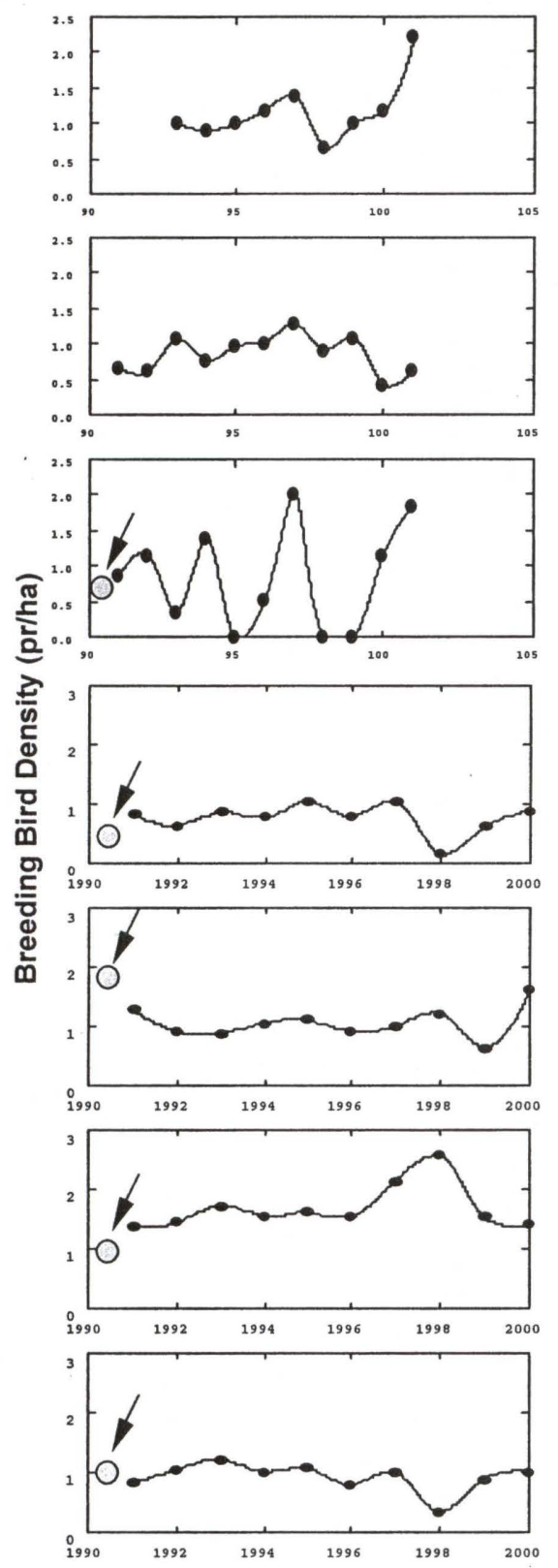

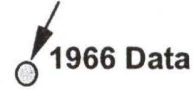

Savannah Sparrow: Site 2

Vesper Sparrow: Site 4

Brewer's Sparrow: Site 4

White-crowned Sparrow: Site 10

Lincoln's Sparrow: Site 10

Song Sparrow: Site 10

Fox Sparrow: Site 10 
Figure 3.

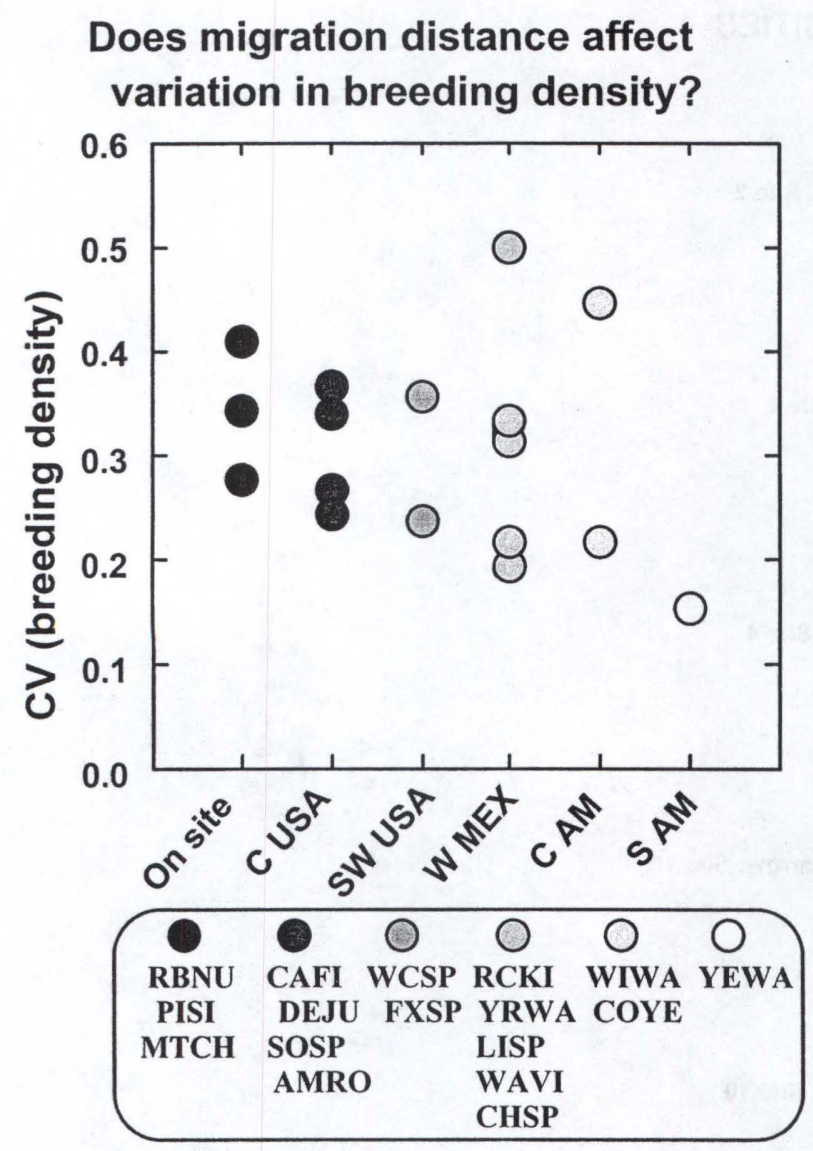


APPENDIX A

\begin{tabular}{|c|c|c|}
\hline GTNP & MONITORING SITES: & SPECIES LIST 1995-2001 \\
\hline PELECANIDAE & White Pelican & Pelecanus erythrorhynchus \\
\hline ARDEIDAE & Great Blue Heron & Ardea herodias \\
\hline GRUIDAE & Sandhill Crane & Grus canadensis \\
\hline \multirow[t]{16}{*}{ ANATIDAE } & Trumpeter Swan & Cygnus buccinator \\
\hline & Canada Goose & Branta canadensis \\
\hline & Mallard & Anas platyrhynchos \\
\hline & Gadwall & Anas strepera \\
\hline & American Wigeon & Anas americana \\
\hline & Cinnamon Teal & Anas cyanoptera \\
\hline & Green-winged Teal & Anas crecca \\
\hline & Blue-winged Teal & Anas discors \\
\hline & Redhead & Aythya americana \\
\hline & Ring-necked Duck & Aythya collaris \\
\hline & Lesser Scaup & Aythya affinis \\
\hline & Common Merganser & Mergus serrator \\
\hline & Barrow's Goldeneye & Bucephala islandica \\
\hline & Common Goldeneye & Bucephala clangula \\
\hline & Bufflehead & Bucephala albeola \\
\hline & Wood Duck & Aix sponsa \\
\hline \multirow[t]{3}{*}{ RALLIDAE } & Virginia Rail & Rallus limicola \\
\hline & Sora & Porzana carolina \\
\hline & American Coot & Fulica americana \\
\hline \multirow[t]{2}{*}{ CHARADRIIDAE } & Kildeer & Charadrius vociferus \\
\hline & Willet & Catoptrophorus semipalmatus \\
\hline \multirow[t]{4}{*}{ SCOLOPACIDAE } & Long-billed Curlew & Numenius americanus \\
\hline & Spotted Sandpiper & Actitis macularia \\
\hline & Wilson's phalarope & Phlaropus tricolor \\
\hline & Common Snipe & Gallinago gallinago \\
\hline \multirow[t]{3}{*}{ LARIDAE } & Franklin's Gull & Larus pipixcan \\
\hline & California Gull & Larus californicus \\
\hline & Ring-billed gull & Larus delawarensis \\
\hline CATHARTIDAE & Turkey Vulture & Cathartes aura \\
\hline \multirow[t]{4}{*}{ ACCIPITRIDAE } & Golden Eagle & Aquila chrysaetos \\
\hline & Bald Eagle & Haliaeetus leucocephalus \\
\hline & Northern Harrier & Circus cyaneus \\
\hline & Red-tailed Hawk & Buteo jamaicensis \\
\hline
\end{tabular}




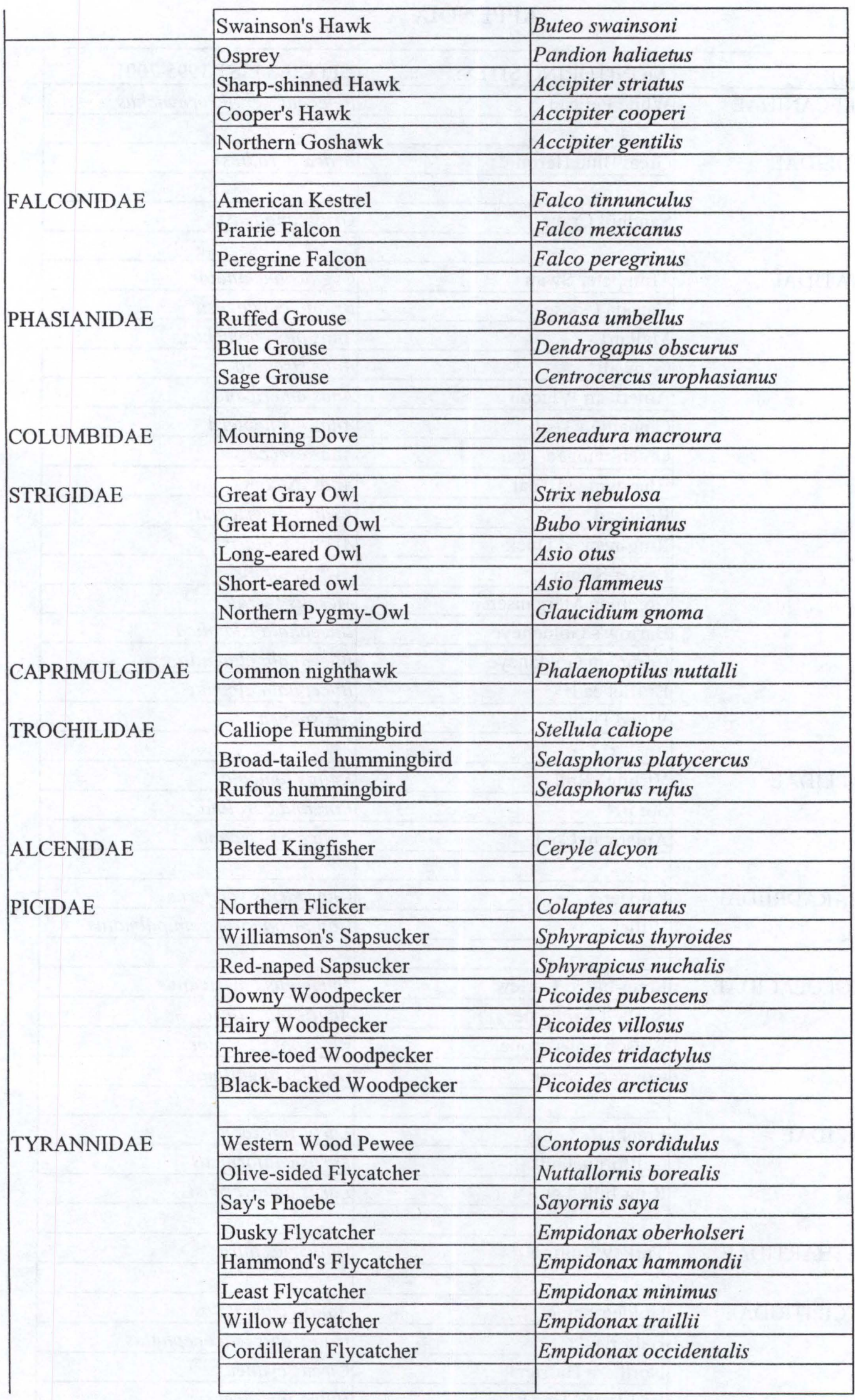




\begin{tabular}{|c|c|c|}
\hline HIRUNDINIDAE & Tree Swallow & Tachycineta bicolor \\
\hline \multirow{11}{*}{ CORVIDAE } & Violet-green swallow & Tachycineta thalassina \\
\hline & Rough-winged Swallow & Stelgidopteryx serripennis \\
\hline & Cliff Swallow & Hirundo pyrrhonota \\
\hline & Barn Swallow & Hirundo rustica \\
\hline & Bank Swallow & Riparia riparia \\
\hline & Steller's Jay & Cyanositta stelleri \\
\hline & Gray Jay & Perisoreus canadensis \\
\hline & Clark's Nutcracker & Nucifraga columniana \\
\hline & Black-billed Magpie & Pica pica \\
\hline & American Crow & Corvus brachyrhynchos \\
\hline & Common Raven & Corvus corax \\
\hline \multirow[t]{2}{*}{ PARIDAE } & Black- capped Chickadee & Parus atricapillus \\
\hline & Mountain Chickadee & Parus gambeli \\
\hline CERTHIDAE & Brown Creeper & Certhia americana \\
\hline \multirow[t]{2}{*}{ SITTIDAE } & White-breasted Nuthatch & Sitta carolinensis \\
\hline & Red-breasted Nuthatch & Sitta canadensis \\
\hline \multirow[t]{3}{*}{ TROGLODYTIDAE } & Marsh Wren & Cistothorus palustris \\
\hline & Rock Wren & Salpinctes obsoletus \\
\hline & House Wren & Troglodytes aedon \\
\hline \multirow{3}{*}{$\begin{array}{l}\text { MUSCICAPIDAE } \\
\text { Sylviinae }\end{array}$} & Golden-crowned Kinglet & Regulus satrapa \\
\hline & Ruby-crowned Kinglet & Regulus calendula \\
\hline & Mountain Bluebird & Sialia currucoides \\
\hline \multirow[t]{5}{*}{ Turdinae } & Townsend's Solitaire & Myadestes townsendi \\
\hline & Veery & Catharus fuscescens \\
\hline & Hermit Thrush & Catharus guttatus \\
\hline & \begin{tabular}{|l} 
Swainson's Thrush \\
\end{tabular} & Catharus ustulatus \\
\hline & American Robin & Turdus migratorius \\
\hline \multirow[t]{2}{*}{ MIMIDAE } & Gray Catbird & Dumatella carolinensis \\
\hline & Sage Thrasher & Oreoscoptes montanus \\
\hline MOTACILLIDAE & Water Pipit & Anthus spinoletta \\
\hline CINCLIDAE & American Dipper & Cinclus mexicanus \\
\hline BOMBYCILLIDAE & Cedar Waxwing & Bombycilla cedrorum \\
\hline STURNIDAE & European Starling & Sturnus vulgaris \\
\hline VIREONIDAE & Solitary Vireo & Vireo solitarius \\
\hline
\end{tabular}




\begin{tabular}{|c|c|c|}
\hline & \multirow{2}{*}{ Warbling Vireo } & \multirow{2}{*}{ Vireo gilvus } \\
\hline & & \\
\hline PASSERIDAE & House sparrow & Passer domesticus \\
\hline \multirow{9}{*}{$\begin{array}{l}\text { EMBERIZIDAE } \\
\text { Parulinae }\end{array}$} & Orange-crowned Warbler & Vermivora celata \\
\hline & Yellow-rumped Warbler & Dendroica coronata \\
\hline & Townsend's warbler & Dendroica townsendi \\
\hline & Yellow Warbler & Dendroica petechia \\
\hline & MacGillivray's Warbler & Oporornis tolmiei \\
\hline & Wilson's Warbler & Wilsonia pusilla \\
\hline & Northern Waterthrush & Seiurus novaboracensis \\
\hline & Common Yellowthroat & Geothlypis trichas \\
\hline & American Redstart & Setophaga ruticilla \\
\hline \multirow[t]{15}{*}{ Emberizinae } & Black-headed Grosbeak & Pheuticus melanocephalus \\
\hline & Lazuli Bunting & Passerina amoena \\
\hline & Spotted towhee & Pipilo maculatus \\
\hline & Green-tailed Towhee & Pipilo chlorurus \\
\hline & Vesper Sparrow & Pooecetes gramineus \\
\hline & Savannah Sparrow & Passerculus sandwichensis \\
\hline & Song Sparrow & Melospiza melodia \\
\hline & Lincoln's Sparrow & Melospiza lincolnii \\
\hline & Fox Sparrow & Passerella iliacus \\
\hline & Lark Sparrow & Chondestes grammacus \\
\hline & Chipping Sparrow & Spizella paserina \\
\hline & Clay-colored Sparrow & Spizella pallida \\
\hline & Brewer's Sparrow & Spizella breweri \\
\hline & Dark-eyed Junco & Junco hyemalis \\
\hline & White-crowned Sparrow & Zonotrichia leucophrys \\
\hline \multirow[t]{8}{*}{ ICTERIDAE } & Bobolink & Dolichonyx oryzivorus \\
\hline & Western Meadowlark & Sturnella neglecta \\
\hline & Yellow-headed Blackbird & Xanthocephalus xanthocephalus \\
\hline & Red-winged Blackbird & Agelaius phoeniceus \\
\hline & Brewer' Blackbird & Euphagus cyanocephalus \\
\hline & Brown-headed Cowbird & Molothru ater \\
\hline & Common Grackle & Quiscalus quiscula \\
\hline & Bullock's Oriole & Icterus bullockii \\
\hline THRAUPIDAE & Western Tanager & Piranga ludoviciana \\
\hline \multirow[t]{6}{*}{ FRINGILLIDAE } & Pine siskin & Carduelis pinus \\
\hline & American Goldfinch & Carduelis tristis \\
\hline & Red Crossbill & Loxia curvirostra \\
\hline & White-winged crossbill & Loxia leucoptera \\
\hline & Pine Grosbeak & Pinicola enucleator \\
\hline & Rosy Finch & Leucosticte arctoa \\
\hline
\end{tabular}




\begin{tabular}{|l|l|l|}
\cline { 2 - 3 } & Cassin's Finch & Carpodacus cassinii \\
\hline & House Finch & Carpodacus mexicanus \\
\hline Evening Grosbeak & Coccothraustes verpertinus \\
\hline & \\
\hline & \\
\hline To Date: 152 species & \\
\hline & \\
\hline
\end{tabular}

